\title{
Time-resolved gas thermometry by laser-induced grating spectroscopy with a high-repetition rate laser system
}

\author{
Felix J. Förster ${ }^{1} \cdot$ Cyril Crua $^{2} \cdot$ Martin Davy $^{3} \cdot$ Paul Ewart $^{1}$
}

Received: 23 March 2017 / Revised: 17 May 2017 / Accepted: 21 May 2017 / Published online: 7 June 2017

(C) The Author(s) 2017. This article is an open access publication

\begin{abstract}
Thermometry using laser-induced grating spectroscopy (LIGS) is reported using a high-repetition rate laser system, extending the technique to allow timeresolved measurements of gas dynamics. LIGS signals were generated using the second harmonic output at $532 \mathrm{~nm}$ of a commercially available high-repetition rate Nd:YAG laser with nitrogen dioxide as molecular seed. Measurements at rates up to $10 \mathrm{kHz}$ were demonstrated under static cell conditions. Transient temperature changes of the same gas contained in a cell subjected to rapid compression by injection of gas were recorded at $1 \mathrm{kHz}$ to derive the temperature evolution of the compressed gas showing temperature changes of $50 \mathrm{~K}$ on a time-scale of $0.1 \mathrm{~s}$ with a measurement precision of $1.4 \%$. The data showed good agreement with an analytical thermodynamic model of the compression process.
\end{abstract}

\section{Introduction}

Gas dynamic processes often take place on time-scales of milliseconds, but the measurement of transient thermodynamic parameters such as temperature presents significant challenges at such time-scales. Measurement difficulties are particularly acute in combusting and non-combusting

Felix J. Förster

felix.foerster@physics.ox.ac.uk

1 Department of Physics, Clarendon Laboratory, University of Oxford, Parks Road, Oxford OX1 3PU, UK

2 Advanced Engineering Centre, University of Brighton, Lewes Road, Brighton BN2 4GJ, UK

3 Department of Engineering Science, University of Oxford, Parks Road, Oxford OX1 3PJ, UK flows where physical probes may prove too invasive and perturbing. Fast-response thermocouples can be used in some situations, but optical techniques are sometimes the only alternative when a non-invasive technique is required. Methods using continuous wave lasers, such as tunable diode laser absorption spectroscopy, can provide temperature data when the absorption line being probed can be scanned at high-repetition rates, but, being a line-of-sight technique, the method lacks spatial resolution (Allen 1998; Davidson et al. 1991; Hanson et al. 1977; Hanson and Davidson 2014). Techniques using pulsed lasers can be limited by the repetition rate of the lasers used-typically $10 \mathrm{~Hz}$ - for a range of techniques including Rayleigh scattering, laser-induced fluorescence, and non-linear methods such as coherent anti-Stokes Raman scattering, CARS. High-speed CARS, using femto-second mode-locked lasers, is an attractive option, but the systems are usually complex and expensive, and require sophisticated data analysis (Eckbreth 1996; Kohse-Höinghaus and Jeffries 2002; Roy et al. 2010). Laser-induced grating spectroscopy (LIGS), also referred to as laser-induced thermal acoustics (LITA), offers an alternative method for gas dynamic measurements with somewhat simpler analysis and relatively simple laser systems.

LIGS relies on the formation of a spatially periodic modulation of the complex refractive index, i.e. a grating, off which a signal beam is generated in a first-order Bragg scattering process. The transient grating is formed by crossing two beams of pulsed laser light to establish the periodic interference pattern in the region of intersection. The spatial period of this pattern is determined by the wavelength of the pump beams and the crossing angle, $\theta$ (usually small). In general, two effects contribute to the grating formation, viz. electrostriction, which is non-resonant, and resonant absorption leading 
to a stationary spatial temperature and density modulation-the thermal grating. When the excitation is rapid, two counter-propagating acoustic waves are generated leading to a periodic modulation of the grating scattering efficiency. The grating evolution is detected by the scattering of a probe beam at the Bragg angle from the induced grating. In the case where the thermal grating dominates, the interaction of the standing acoustic wave with the stationary grating leads to a scattered signal in the form of a decaying sinusoidal oscillation at a frequency determined by the local speed of sound. The form of the signal, its intensity, modulation amplitude, and decay are related to the collisional quenching rates of the molecular species involved and the thermal diffusion and acoustic damping rates. The signal parameters provide information on the temperature (through the local speed of sound) and pressure (from the signal damping rate). In addition, the signal intensity is related to the absorber concentration and the Doppler shifts of the scattered signal give information on the bulk gas velocity. The LIGS signal, therefore, may be analysed to give information on multiple parameters simultaneously: temperature (Cummings 1994; Latzel et al. 1998), pressure (Stevens and Ewart 2004; Hart et al. 2007), flow velocity (Mach number) (Walker et al. 1998; Kozlov 2005; Hemmerling et al. 2000), and concentration of the absorbing species (Seeger et al. 2006; Roshani et al. 2013). A particular advantage of LIGS is the robustness and high precision of temperature measurements that results from the measuring of a frequency rather than intensity (Stevens and Ewart 2004; Williams et al. 2014; Förster et al. 2015). Applications of LIGS, however, have so far been limited to measurements at the $10 \mathrm{~Hz}$ repetition rate of the high-energy, pulsed lasers used (Kiefer and Ewart 2011).

Although LIGS has already been used to measure parameters during transient phenomena, the ability to measure changes at higher repetition rates will significantly improve the study of inherently fast phenomena such as combustion in high-speed flows (Hell et al. 2016) and mixing processes involved in high-pressure fuel injection (Baab et al. 2016). In addition, measurements will become potentially possible for other parameters such as turbulence intensity in unsteady flows as well as combustion dynamics.

In this paper, we show that such measurements can now, in principle, be made at high-repetition rates allowing rapid changes in thermodynamic properties to be recorded with high precision. Hence, high-speed or high-repetition rate LIGS offers significant potential benefits for applications to rapidly changing environments or transient effects in combusting and non-combusting flows.

\section{Equipment and optical setup}

The key requirements for the laser sources for LIGS are that the pump laser pulses contain enough energy to generate sufficient perturbation of the medium's refractive index providing adequate scattering efficiency from the induced grating. However, it is also important to ensure that the temperature is not appreciably perturbed by the measurement process itself (Kiefer and Ewart 2011). Of critical importance is that the pulse duration is short relative to the transit time of the induced acoustic waves to cross the fringe spacing of the grating. In practice, these conditions are usually satisfied with pulses of the order of a few $\mathrm{mJ}$ and duration $\approx 5-10 \mathrm{~ns}$. In addition, the beam quality needs to be good enough, so that the transverse coherence provides good contrast in the induced grating with spatially uniform intensity. Good spatial uniformity and low divergence are also important for the probe laser, and in practice, a power level of the order of 300$1000 \mathrm{~mW}$ is usually adequate. For optimum efficiency, it is also useful to match the cross-sectional area of both the pump and the probe beams.

The high-repetition rate in the present experiments was enabled by the use of a pulsed Nd:YAG laser designed for high-speed PIV (Particle Image Velocimetry) measurements (Edgewave InnoSlab Model IS 16II). Such lasers are becoming increasingly available commercially for PIV studies and other high-speed imaging applications. The second harmonic output at $532 \mathrm{~nm}$ was used to match the absorption in nitrogen dioxide $\left(\mathrm{NO}_{2}\right)$ that was used as the absorbing medium. Pulse repetition rates between $10 \mathrm{~Hz}$ and $10 \mathrm{kHz}$ were used in which individual pulses had a duration of $9 \mathrm{~ns}$ and an energy of $8 \mathrm{~mJ}$ per pulse. The output beam had a rectangular spatial profile, $3 \times 8 \mathrm{~mm}$, with an approximately Gaussian intensity distribution across the short dimension. Hence, this laser provides the beam quality required for the technique. An additional advantage is that the rectangular output would make this laser particularly suited to 1-D LIGS measurements as reported recently (Willman and Ewart 2016).

A continuous wave probe beam was provided by a diode-pumped solid-state laser (Laser Quantum, Ventus) emitting light at $660 \mathrm{~nm}$ with a power of $750 \mathrm{~mW}$. The beam from the high-repetition rate laser was split by a 50:50 beam splitter to form two pump pulses propagating in parallel and separated by $36 \mathrm{~mm}$. The pump and probe beams were focused in the front focal plane of a $500 \mathrm{~mm}$ focal length lens, such that they crossed as collimated beams in the back focal plane with beam diameters approximately $1 \mathrm{~mm}$ in diameter to give an interaction length of approximately $20 \mathrm{~mm}$. No change in the geometry of the interaction volume was observed when the pulse repetition rate was changed. This arrangement produced acoustic 
oscillations of the LIGS signal at a frequency of approximately $48 \mathrm{MHz}$ using $\mathrm{NO}_{2}$ as the absorber in a buffer gas of $\mathrm{N}_{2}$ at ambient conditions. The resulting LIGS signal was directed along a $2 \mathrm{~m}$ path to a photomultiplier for detection and recording on a $2.5 \mathrm{GHz}$ bandwidth digital oscilloscope (LeCroy Waverunner 625Zi).

The initial tests of the system were carried out under static conditions. $\mathrm{NO}_{2}$ was admitted to an initially evacuated cell to provide a final concentration of $5000 \mathrm{ppm}$ when filled with $\mathrm{N}_{2}$ at 4 bar pressure and allowed to reach thermal equilibrium at ambient temperature. Figure 1a shows part of the continuous recording of the signal at a repetition rate of $10 \mathrm{kHz}$ in which five consecutive signals are observed as sharp "spikes" on the oscilloscope trace covering $0.5 \mathrm{~ms}$. These data are shown only to illustrate the high-repetition rate of the system and is clearly not optimum for data acquisition with sufficient resolution to record the time behaviour of the LIGS signals. Two individual LIGS signals are shown, however, on a faster time base in Fig. 1b, such that the typical signal shape and the acoustic oscillations are clearly resolved.

The speed of sound may be derived from the experimental LIGS signal in one of several possible analytical approaches. In some cases, with signals of adequate quality, the acoustic oscillation frequency can be obtained by a Fourier transform of the signal intensity as a function of time (Balla and Miller 2008; Förster et al. 2015). Alternatively, a model signal may be fitted to the experimental data using the speed of sound as a fitting parameter (Cummings et al. 1995; Paul and Farrow 1995; Stevens and Ewart 2004). In the present work, the modelled signals, fitted to the data, were calculated using the theoretical model based on the linearized hydrodynamic equations of reference (Cummings et al. 1995). A typical, single-shot, signal recorded at $10 \mathrm{kHz}$ is shown in Fig. 2 together with the computed "best-fit" model signal.
The precision of the measurements, indicated by the standard deviation of 100 sample signals recorded for static conditions, is shown in Table 1 for repetition rates between $10 \mathrm{~Hz}$ and $10 \mathrm{kHz}$. The decrease in precision with increasing repetition rate is due to the decreasing signal-to-noise ratio resulting from lower pulse energies at the higher rates. However, the measured uncertainties are still in the range expected for the technique of less than $1 \%$. The temperature is derived from the speed of sound, typically either via a thermodynamic properties database or-for small changes in temperature and pressure, as in the present work-directly from the ideal gas law in the form of $T=c_{\mathrm{s}}^{2} / R \gamma$, where $c_{\mathrm{S}}$ is the speed of sound, $R$ is the gas constant, and $\gamma$ is the ratio of specific heats at constant volume and pressure. Given the small concentration of $\mathrm{NO}_{2}$ in the $\mathrm{N}_{2}$ and at the temperature and pressure studied in this work, a constant value of $\gamma$ is assumed and taken to have the value $\gamma=1.403$ determined by the gas composition. With these assumptions, the standard deviation in temperature values derived from

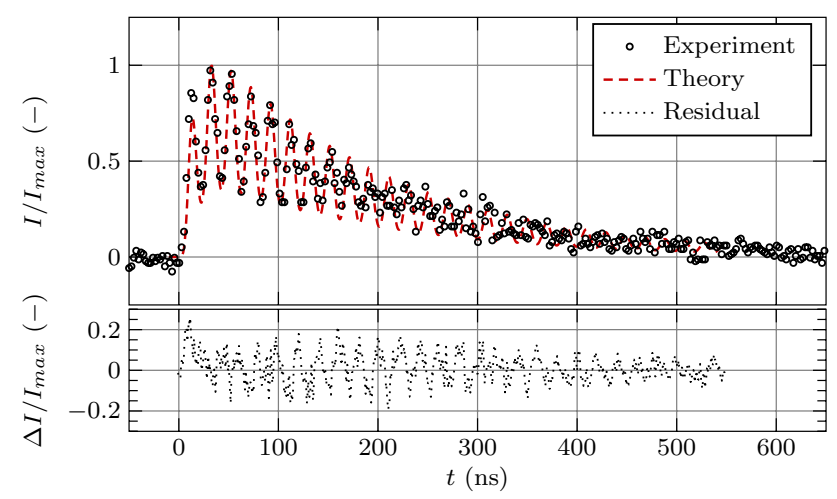

Fig. 2 Recorded and fitted signal together with the residual taken at $10 \mathrm{kHz}$
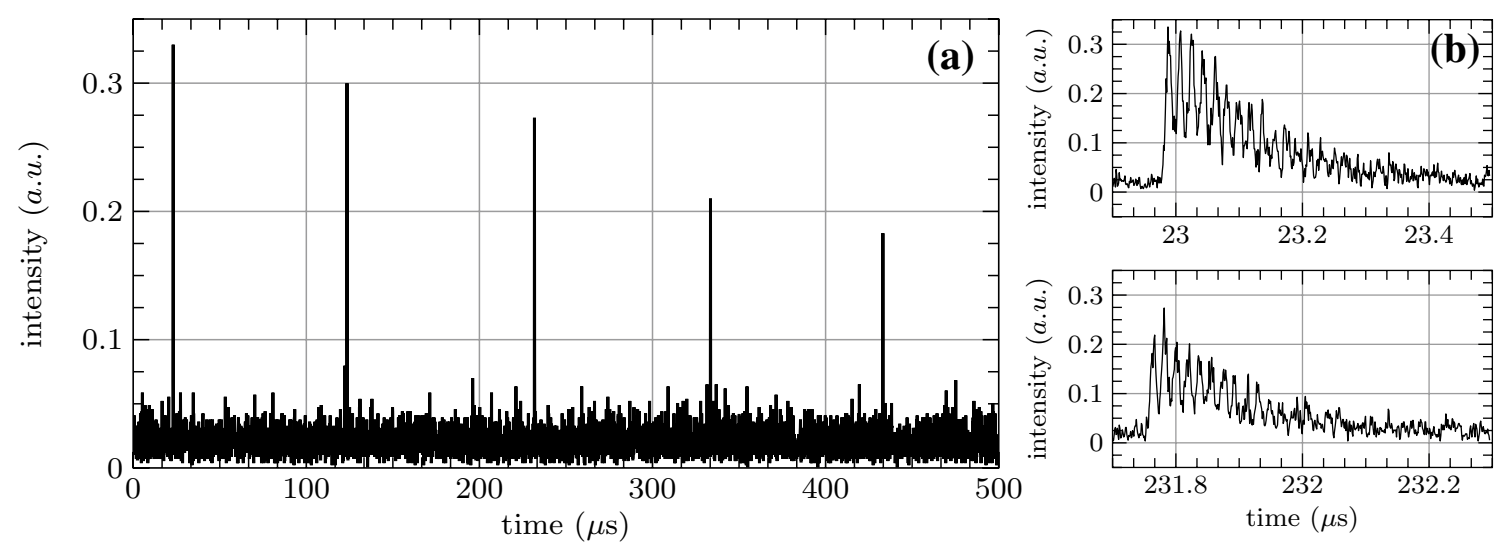

Fig. 1 a Continuous recording of LIGS signals generated $10 \mathrm{kHz}$. b Two individual LIGS signals, lasting approximately $300 \mathrm{~ns}$, shown on an expanded time-scale 
Table 1 Comparison of the relative standard deviation in the speed of sound based on 100 samples and signal-to-noise ratios for different repetition rates

\begin{tabular}{lllll}
\hline Repetition rate & $10 \mathrm{~Hz}$ & $100 \mathrm{~Hz}$ & $1 \mathrm{kHz}$ & $10 \mathrm{kHz}$ \\
Rel. standard deviation & $0.1 \%$ & $0.2 \%$ & $0.3 \%$ & $0.7 \%$ \\
SNR & 62 & 53 & 36 & 17 \\
\hline
\end{tabular}

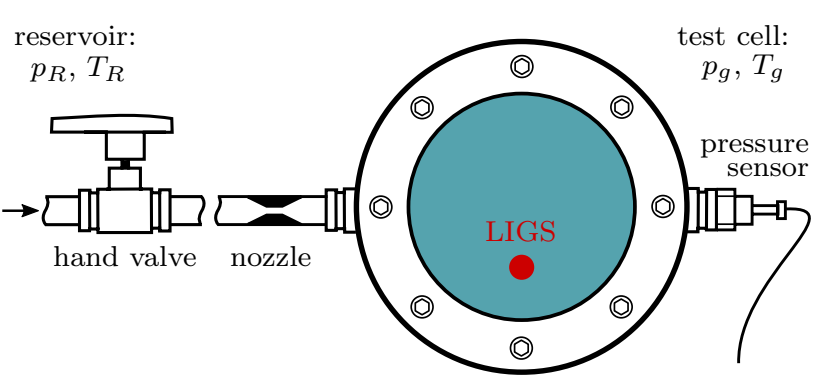

Fig. 3 Setup of the transient experiment: the location of the LIGS measurement volume is indicated by the red circle

the speed of sound will simply be twice the values quoted for the speed of sound in Table 1.

\section{Transient flow experiment}

In addition to the measurements under static conditions, a more intuitive demonstration of the high-speed capability is given in an experiment to study a transient effect. The experiment is designed to provide a well-defined, yet fast temperature rise as result of the compression of a test gas: a scenario found, for instance, in the refuelling of a gaseous fuel tank. Figure 3 shows the arrangement for this experiment. A reservoir containing $\mathrm{NO}_{2}$ gas at 5 bar pressure is connected via a ball valve and a nozzle to a test cell, of volume $30 \mathrm{ml}$, containing a well-mixed gas consisting of $5000 \mathrm{ppm} \mathrm{NO} \mathrm{N}_{2}$ in $\mathrm{N}_{2}$ at ambient pressure $(\approx 1$ bar $)$ and temperature $(\approx 280 \mathrm{~K})$. A fast-response pressure transducer monitored the pressure in the cell. A rapid change in pressure (and thus temperature) in the cell was initiated by rapid manual opening of the ball valve. The rate at which the transient changes in pressure and temperature in the cell occur is limited by the geometric restriction of the nozzle. As it will be shown in Sect. 3.2, the restriction determined by the nozzle throat diameter $(1 \mathrm{~mm})$ sets the time-scale of the experiment, such that a few data points could be obtained during the transient phase even using a repetition rate of $10 \mathrm{~Hz}$. Measurements at $10 \mathrm{~Hz}$ provided a baseline reference for comparison with the previous experiments using LIGS with this repetition rate where the technique is known to be accurate. Although measurements at a higher rate will show the temperature evolution in more detail, the
$10 \mathrm{~Hz}$ data are adequate to provide a general validation of the high-speed approach. After opening the ball valve, the gas in the cell was allowed to stabilize before being evacuated, re-filled with the $\mathrm{N}_{2} / \mathrm{NO}_{2}$ mixture, and allowed to reach equilibrium before the next test.

LIGS signals were recorded using the oscilloscope which was triggered at the repetition rates of the pump laser, i.e. $10,50,100$, and $1000 \mathrm{~Hz}$, with the measurements at $1 \mathrm{kHz}$ being sufficiently fast to resolve the observed transient temperature changes. The individual signals were recorded over a time of about 600 ns, as shown in Fig. 2. The reading of the pressure transducer was recorded simultaneously over this time interval and at the same repetition rate as the laser. The data acquisition was activated by a signal from the transducer corresponding to the cell pressure reaching 1.2 bar. The delay after initiating the valve opening was found to be reproducible and around $80 \mathrm{~ms}$.

\subsection{Experimental data}

The results of the simultaneous measurements of pressure, sampled from the pressure transducer signal during each laser pulse, and temperature derived from the LIGS signal generated, are shown in Fig. 4. Since the reservoir volume, maintained at a constant pressure of 5 bar, is much larger than that of the cell, the pressure in the cell rises rapidly until it reaches that of the reservoir upstream of the nozzle and stabilizes at that value. This process is shown in Fig. 4a. The identity of all the pressure traces, recorded in a sequence of experiments, shows that the experimental conditions produced by the valve opening are also reproducible and, hence, data recorded at different repetition rates at different times can be compared. In a similar way, the change in temperature associated with this pressure rise in the constant volume test cell is shown in Fig.4b for the different repetition rates of the laser. The data recorded at $10 \mathrm{~Hz}$ represent the baseline measurement, since the technique is known to be accurate and precise at this repetition rate.

The temporal profile of the temperature change is characterised by a steep initial rise as the pressure increases in the cell. The temperature reaches a reproducible maximum within approximately $200 \mathrm{~ms}$ and then decreases approximately exponentially back to the ambient level, as a result of heat dissipation, after approximately $5 \mathrm{~s}$. As shown by the data, the temporal behaviour is reproduced for all repetition rates of the LIGS measurement system, showing that the technique is as accurate in the high-repetition rate mode as in the baseline case.

Figure 5 shows the initial rise in pressure and temperature on an expanded time base. The benefit of fast, i.e. highrepetition rate, measurements is clearly seen in these data. Although some data points are acquired at $10 \mathrm{~Hz}$ within the initial rise, it is only the $1000 \mathrm{~Hz}$ data that provide fully 

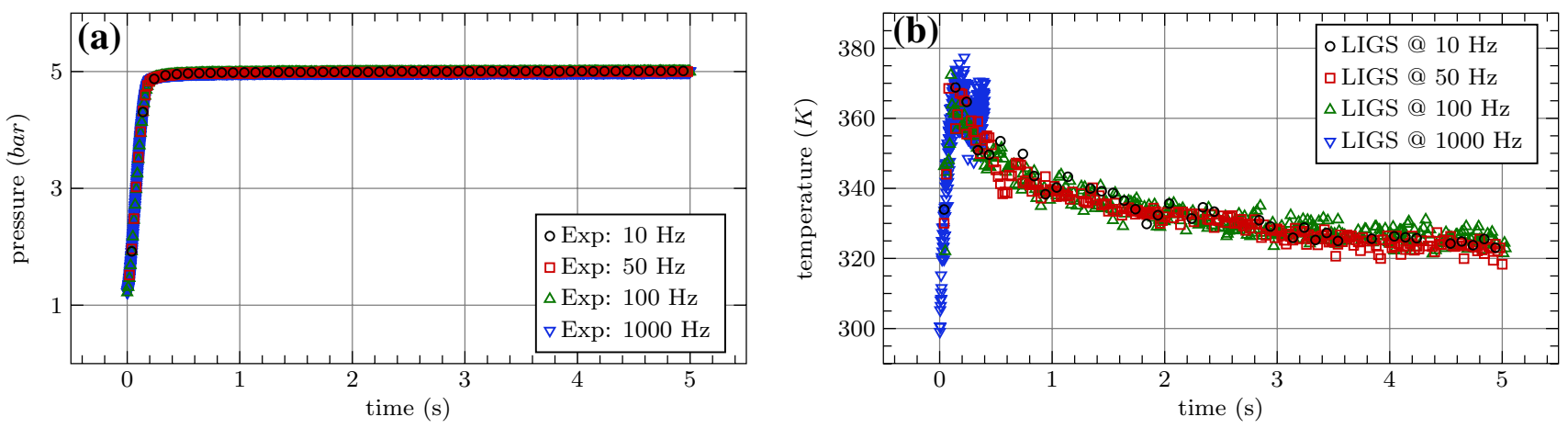

Fig. 4 a Pressure and $\mathbf{b}$ temperature traces: long-term behaviour
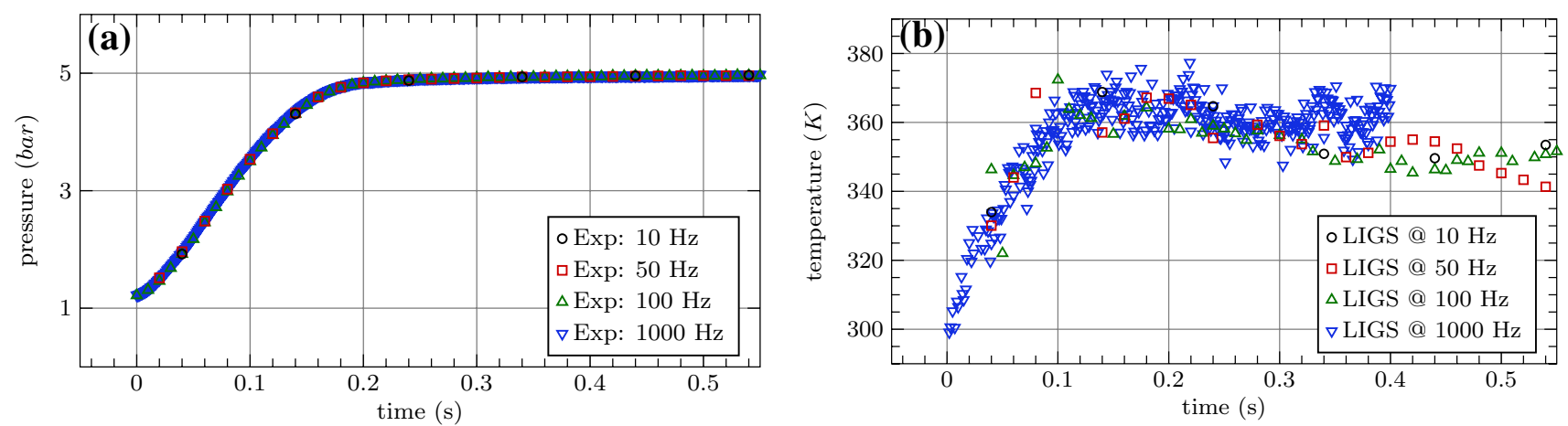

Fig. 5 a Pressure and $\mathbf{b}$ temperature traces: initial temperature rise

time-resolved measurements. At this higher recording rate, fluctuations or oscillations become apparent. Such fluctuations may arise from the compressible nature of the gas in the system and would not be visible at lower repetition rates. It is possible, even likely, that these oscillations arise from reflection of the perturbation waves with a frequency that depends on the dimensions of the apparatus.

\subsection{Thermodynamic model}

To confirm the validity of these measurements, comparison is made with predictions from an analytical model to show that the values derived from the data are within the expected range regarding the rise time and absolute change in temperature. The model makes the following simplifying assumptions. The volume of the test cell is considered constant and defines the control volume within which energy is conserved. Changes in potential energy between the reservoir and test cell are neglected and the process is assumed to be adiabatic, i.e. there is no heat loss to the cell walls on the time-scale of the pressure change induced by opening the valve. The change in total energy in the cell is then determined solely by the mass flow entering the cell and the specific enthalpy of the gas entering the cell. For an adiabatic flow between test cell and reservoir, the specific total enthalpy of the gas along the pipe is constant and, therefore, equal to the reservoir conditions. As the reservoir volume is effectively large compared to that of the cell, the gas properties in the reservoir suffer negligible change during the process. The total energy of the gas in the cell, however, is given by the product of the mass of gas in the cell and the specific internal energy provided that all the kinetic energy of the incoming gas is converted to internal energy of the gas in the cell. Assuming, further, that nitrogen behaves as a calorically perfect gas, the specific enthalpy and internal energy are functions only of temperature. Under these conditions, the temperature of the gas in the cell, as a function of time, $T_{\mathrm{g}}(t)$, can be expressed as follows:

$\frac{T_{\mathrm{g}}(t)-\gamma T_{\mathrm{r}}}{T_{0}-\gamma T_{\mathrm{r}}}=\frac{m_{0}}{m(t)}$

where $T_{\mathrm{r}}$ is the temperature in the reservoir, $m(t)$ the mass in the cell at time $t$, and $m_{0}$ the initial mass. As discussed above, $\gamma$ was set to be constant and equal to 1.403. Applying the ideal gas law for a constant volume, the analogous expression for pressure is as follows:

$\frac{p_{\mathrm{g}}(t)-p_{0}}{p_{0}}=\gamma \frac{m(t)-m_{0}}{m_{0}}$ 
where $p_{\mathrm{g}}(t)$ is the gas pressure in the cell at time $t$ and $p_{0}$ is the initial pressure.

Equations 1 and 2 show that for given initial reservoir and cell conditions, the evolution of temperature and pressure in the cell is a function of the mass added over time. The mass flow in the connection pipe may be treated as one-dimensional and is constant along the pipe. Hence, it only depends on the thermodynamic conditions in the reservoir and cell as well as the pipe geometry. The ball valve opening is sufficiently rapid that the mass flow is effectively controlled by the nozzle geometry and its aperture will determine the rate at which mass is added to the cell. In addition, the mass flow will be constant for such choked flow conditions transforming Eqs. 1 and 2 into the firstorder functions of time.

Figure 6 compares the experimental and theoretical pressure traces in which three regimes are identified. The regime where the pressure rise linearly corresponds to a fully open valve and choked flow conditions (regime 2). In this case, the calculated pressure (dashed line) is in good agreement with the recorded pressure data (dots), but deviations occur outside this regime. In the initial phase (regime 1), the discrepancy is due to the finite opening time of the valve, such that initially, the smallest orifice is the valve rather than the nozzle. As choked flow conditions still apply, this could be modelled by varying the cross-sectional area as a function of time to reflect the opening characteristics of the valve. An expression for mass flow through the ball valve, $m(t)$, was approximated by calculating the rate at which the opening aperture changed, assuming that it is defined by the overlap of the circular orifice in the rotating ball with the circular pipe aperture and the valve goes from fully closed to fully open in the $80 \mathrm{~ms}$ time observed empirically.

The situation is more complex for regime 3 , since the cell pressure approaches that of the reservoir and choked flow conditions no longer apply. In this case, the mass flow becomes dependent on the reservoir pressure, $p_{\mathrm{r}}$, as well as

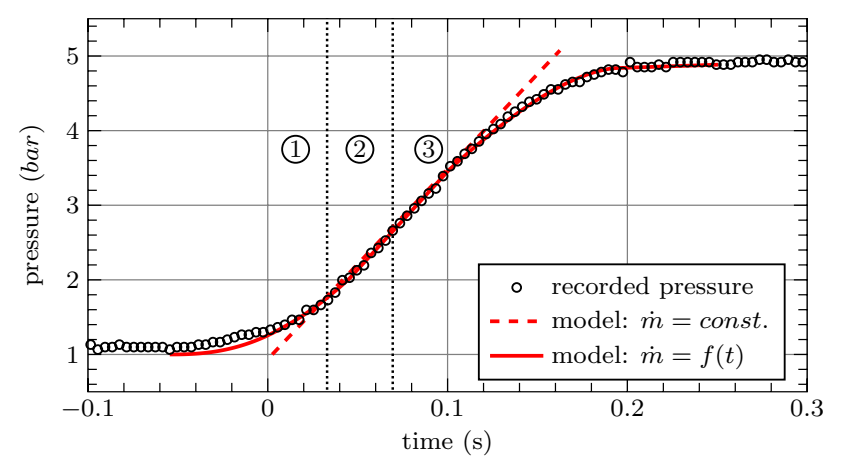

Fig. 6 Pressure obtained from the analytical model and the pressure transducer the instantaneous downstream pressure, $p_{\mathrm{g}}$, which is a priori unknown and will depend on the flow velocity, or Mach number, $M_{\text {exit }}$, through the nozzle of cross-sectional area $A_{\text {nozzle }}$. The mass flow, or rate of change of mass in the cell, $\dot{m}$, is given in this case by the following:

$$
\begin{gathered}
\frac{p_{\mathrm{g}}}{p_{\mathrm{r}}}=\left(1+\frac{\gamma-1}{2} M_{\text {exit }}^{2}\right)^{-\frac{\gamma}{\gamma-1}} \\
\dot{m}=\frac{A_{\text {nozzle }}}{2 R T_{\mathrm{r}}} M_{\text {exit }} \sqrt{\frac{2 \gamma T_{\mathrm{r}}}{\gamma+1}}(\gamma+1) p_{\mathrm{r}} \\
\quad \times\left(\frac{1}{2}+\frac{\gamma}{2}\right)^{-\frac{\gamma}{\gamma-1}}\left(\frac{2+\gamma M_{\text {exit }}^{2}-M_{\text {exit }}^{2}}{\gamma+1}\right)^{\frac{-(\gamma+1)}{2(\gamma-1)}} .
\end{gathered}
$$

The flow rate and, hence, the pressure in the cell can be found by iteration of Eqs. 2, 3, and 4. In practice, an acceptable fit to the data, i.e. within experimental error, was obtained after only three iterations.

Thus, the original model was modified to account for the three phases of the compression process corresponding to three regimes: (1) choked flow with varying nozzle throat, (2) choked flow with constant nozzle throat, and (3) unchoked flow using an iterative solution. As shown in Fig. 6, this solution for the time-depending mass flow (solid line) matches closely the pressure trace recorded by the pressure transducer.

This validation permits using the same mass flow evolution to calculate the temperature as function of time according to Eq. 1. Figure 7 presents a comparison of the model's prediction with the temperature derived from LIGS measurements at $1 \mathrm{kHz}$ and shows reasonable agreement for both the rise time and overall increase in temperature between the calculated and measured profiles. The model over-predicts the temperature rise and this is presumably because heat losses to the walls and cell windows were

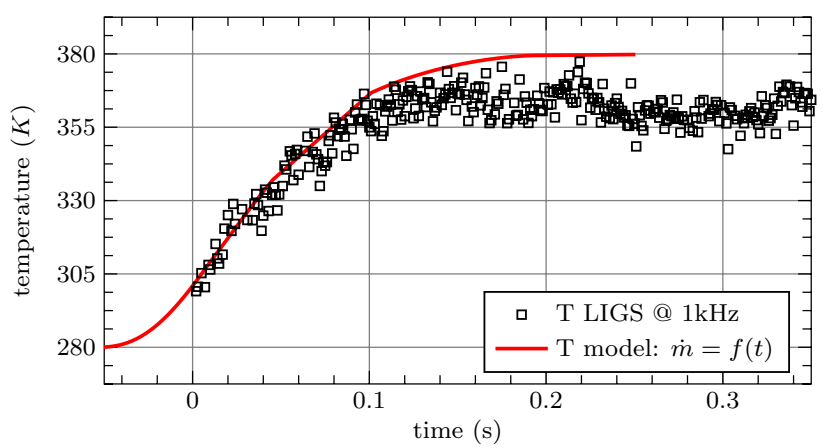

Fig. 7 Temperature obtained from the analytical model and the LIGS technique 
neglected. The same reason accounts for the increasing discrepancy found after the peak temperature is reached, since the absence of losses in the idealized model results in a constant elevated gas temperature. The experimental temperature data, derived from the LIGS measurements, show some oscillatory behaviour, or "ringing", which originates from the more complex flow dynamics associated with the particular geometry of the valve, pipe, and cell assembly used that are not represented in the model.

\section{Conclusions}

This work has demonstrated, for the first time, that LIGS is capable of temperature measurement at rates of up to $10 \mathrm{kHz}$ using a high-repetition rate pump laser. The improvement, by up to a factor of $10^{3}$, in the time resolution overcomes the significant limitation, imposed by $10 \mathrm{~Hz}$ systems, for studies of transient gas dynamic processes and flow diagnostics. Measurement precision for the speed of sound was found, in static conditions, to be in the range of $0.1-1 \%$ over the range of repetition rates from $10 \mathrm{~Hz}$ to $10 \mathrm{kHz}$, at ambient temperatures and for pressures up to 4 bar. In addition, high-speed thermometry is demonstrated for a transient temperature change. The temperature changes, associated with the rapid compression induced by injection of gas at higher pressure, were resolved using sequential single-shot measurements at up to $1 \mathrm{kHz}$. Measurements at each of the repetition rates were in agreement and in particular with the baseline measurements at $10 \mathrm{~Hz}$. However, only the repetition rate of $1 \mathrm{kHz}$ offered sufficient time resolution for the initial and very sharp temperature increase. This initial phase of the experiment was also described with a thermodynamic model. Its predicted pressure and temperature evolutions yielded good agreement with both the recorded data from the pressure transducer and the LIGS technique, respectively. This provided further validation of the high-speed approach showing that rise time and change in the experiment are captured correctly by the LIGS technique.

In conclusion, this work has demonstrated a significant improvement in the time resolution obtainable using LIGS for thermometry making it applicable to transient conditions such as turbulent flows in many engineering applications. The present demonstration of principle was applied to thermometry, but it is worth noting that the same technique allows simultaneous measurement of other flow parameters such as pressure and flow velocity. The technique, therefore, has potential to measure all three parameters, temperature, pressure, and flow velocity, simultaneously with time and space resolution. The present work used the available wavelength to match the absorption in a convenient medium, $\mathrm{NO}_{2}$, but other wavelengths are also available from the fundamental output or higher harmonics of the Nd:YAG laser to match other absorbers, e.g. aromatic hydrocarbons and ketones in the UV or alkanes in the NIR that might be more suitable as tracers in the desired application.

Acknowledgements This work was funded in part by the Engineering and Physical Sciences Research Council (EPSRC), UK, under Grant Number EP/M009424/1. The authors would also like to thank Dr. G. De Sercey for his technical support.

Open Access This article is distributed under the terms of the Creative Commons Attribution 4.0 International License (http://creativecommons.org/licenses/by/4.0/), which permits unrestricted use, distribution, and reproduction in any medium, provided you give appropriate credit to the original author(s) and the source, provide a link to the Creative Commons license, and indicate if changes were made.

\section{References}

Allen MG (1998) Diode laser absorption sensors for gasdynamic and combustion flows. Meas Sci Technol 9(4):545. doi:10.1088/0957-0233/9/4/001

Baab S, Förster FJ, Lamanna G, Weigand B (2016) Speed of sound measurements and mixing characterization of underexpanded fuel jets with supercritical reservoir condition using laserinduced thermal acoustics. Exp Fluids 57(11):172. doi:10.1007/ s00348-016-2252-3

Balla RJ, Miller CA (2008) Signal analysis algorithms for optimized fitting of nonresonant laser induced thermal acoustics damped sinusoids. Tech. rep, Nasa TechReport, pp 2008-215327

Cummings EB (1994) Laser-induced thermal acoustics: simple accurate gas measurements. Opt Lett 19:1361. doi:10.1364/ OL.19.001361

Cummings EB, Leyva IA, Hornung HG (1995) Laser-induced thermal acoustics (LITA) signals from finite beams. Appl Opt 34(18):3290. doi:10.1364/AO.34.003290

Davidson DF, Chang AY, DiRosa MD, Hanson RK (1991) Continuous wave laser absorption techniques for gasdynamic measurements in supersonic flows. Appl Opt 30(18):2598. doi:10.1364/ ao.30.002598

Eckbreth AC (1996) Laser diagnostics for combustion temperature and species, vol 3. CRC Press, Boca Raton

Förster FJ, Baab S, Lamanna G, Weigand B (2015) Temperature and velocity determination of shock-heated flows with non-resonant heterodyne laser-induced thermal acoustics. Appl Phys B 121(3):235-248. doi:10.1007/s00340-015-6217-7

Hanson RK, Kuntz PA, Kruger CH (1977) High-resolution spectroscopy of combustion gases using a tunable IR diode laser. Appl Opt 16(8):2045. doi:10.1364/ao.16.002045

Hanson R, Davidson D (2014) Recent advances in laser absorption and shock tube methods for studies of combustion chemistry. Prog Energy Combust Sci 44:103. doi:10.1016/j. pecs.2014.05.001

Hart RC, Herring GC, Balla RJ (2007) Pressure measurement in supersonic air flow by differential absorptive laser-induced thermal acoustics. Opt Lett 32(12):1689. doi:10.1364/ol.32.001689

Hell A, Förster FJ, Weigand B (2016) Validation of laser-induced thermal acoustics for chemically reacting $\mathrm{H} 2$ /air free jets. J Raman Spectrosc 47(9):1157. doi:10.1002/jrs.4859

Hemmerling B, Kozlov DN, Stampanoni-Panariello A (2000) Temperature and flow-velocity measurements by use of laser-induced 
electrostrictive gratings. Opt Lett 25(18):1340. doi:10.1364/ ol.25.001340

Kiefer J, Ewart P (2011) Laser diagnostics and minor species detection in combustion using resonant four-wave mixing. Prog Energy Combust Sci 37(5):525. doi:10.1016/j.pecs.2010.11.001

Kohse-Höinghaus K, Jeffries JB (2002) Applied combustion diagnostics. Taylor and Francis, New York

Kozlov DN (2005) Simultaneous characterization of flow velocity and temperature fields in a gas jet by use of electrostrictive laserinduced gratings. Appl Phys B 80:377

Latzel H, Dreizler A, Dreier T, Heinze J, Dillmann M, Stricker W, Lloyd GM, Ewart P (1998) Thermal grating and broadband degenerate four-wave mixing spectroscopy of $\mathrm{OH}$ in high-pressure flames. Appl Phys B 67:667

Paul PH, Farrow RL (1995) Gas-phase thermal-grating contributions to four-wave mixing. J Opt Soc Am B 12:384. doi:10.1364/ JOSAB.12.000384

Roshani B, Flügel A, Schmitz I, Kozlov DN, Seeger T, Zigan L, Kiefer J, Leipertz A (2013) Simultaneous measurements of fuel vapor concentration and temperature in a flash-boiling propane jet using laser-induced gratings. J Raman Spectrosc 44(10):1356. doi: $10.1002 /$ jrs. 4315
Roy S, Gord JR, Patnaik AK (2010) Recent advances in coherent antiStokes Raman scattering spectroscopy: fundamental developments and applications in reacting flows. Progress Energy Combust Sci 36(2):280. doi:10.1016/j.pecs.2009.11.001

Seeger T, Kiefer J, Weikl M, Leipertz A, Kozlov D (2006) Timeresolved measurement of the local equivalence ratio in a gaseous propane injection process using laser-induced gratings. Opt Express 14(26):12994. doi:10.1364/oe.14.012994

Stevens R, Ewart P (2004) Single-shot measurement of temperature and pressure using laser-induced thermal gratings with a long probe pulse. Appl Phys B 78(1):111. doi:10.1007/ s00340-003-1282-8

Walker DJW, Williams RB, Ewart P (1998) Thermal grating velocimetry. Opt Lett 23(16):1316. doi:10.1364/ol.23.001316

Williams B, Edwards M, Stone R, Williams J, Ewart P (2014) High precision in-cylinder gas thermometry using laser induced gratings: quantitative measurement of evaporative cooling with gasoline/alcohol blends in a GDI optical engine. Combust Flame 161(1):270. doi:10.1016/j.combustflame.2013.07.018

Willman C, Ewart P (2016) Multipoint temperature measurements in gas flows using 1-D laser-induced grating scattering. Exp Fluids 57(12):191. doi:10.1007/s00348-016-2282-x 